\title{
How Long Non-Coding RNAs and MicroRNAs Mediate the Endogenous RNA Network of Head and Neck Squamous Cell Carcinoma: a Comprehensive Analysis
}

\author{
Guomiao Zhao Yaru Fu $^{\mathrm{a}}$ Zhifang Su ${ }^{\mathrm{a}}$ Rongling Wu $\mathrm{Wu}^{\mathrm{a}, \mathrm{b}}$ \\ aCenter for Computational Biology, College of Biological Sciences and Technology, Beijing Forestry \\ University, Beijing, China, 'Department of Public Health Sciences, The Pennsylvania State University, \\ Hershey, USA
}

\section{Key Words}

IncRNA • miRNA • Endogenous RNA network • Head and neck squamous cell carcinoma

\begin{abstract}
Background/Aims: Long non-coding RNAs (IncRNAs) act as competing endogenous RNAs (ceRNAs) to compete for microRNAs (miRNAs) in cancer metastasis. Head and neck squamous cell carcinoma (HNSCC) is one of the most common human cancers and rare biomarkers could predict the clinical prognosis of this disease and its therapeutic effect. Methods: Weighted gene co-expression network analysis (WGCNA) was performed to identify differentially expressed mRNAs (DEmRNAs) that might be key genes. GO enrichment and protein-protein interaction (PPI) analyses were performed to identify the principal functions of the DEmRNAs. An IncRNA-miRNA-mRNA network was constructed to understand the regulatory mechanisms in HNSCC. The prognostic signatures of mRNAs, miRNAs, and IncRNAs were determined by Gene Expression Profiling Interactive Analysis (GEPIA) and using Kaplan-Meier survival curves for patients with lung squamous cell carcinoma. Results: We identified 2,023 DEmRNAs, 1,048 differentially expressed IncRNAs (DElncRNAs), and 82 differentially expressed miRNAs (DEmiRNAs). We found that eight DEmRNAs, 53 DElncRNAs, and 16 DEmiRNAs interacted in the ceRNA network. Three ceRNAs (HCG22, LINC00460 and STC2) were significantly correlated with survival. STC2 transcript levels were significantly higher in tumour tissues than in normal tissues, and the STC2 expression was slightly upregulated at different stages of HNSCC. Conclusion: LINC00460, HCG22 and STC2 exhibited aberrant levels of expression and may participate in the pathogenesis of HNSCC.




\section{Cellular Physiology Cell Physiol Biochem 2018;50:332-341

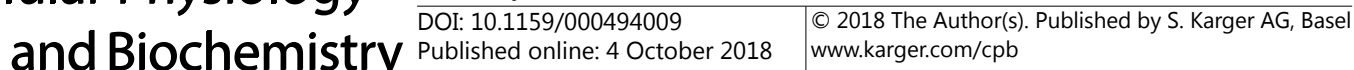 \\ Zhao et al.: Non-Coding RNAs and MicroRNAs Medicate Cancer Cell Network}

\section{Introduction}

Head and neck squamous cell carcinoma (HNSCC), the sixth most common human cancer, is characterised by etiological, phenotypic, biological, and clinical heterogeneity [1]. Many HNSCC patients eventually develop metastatic recurrence [2], and half of all patients die within the 5 years after diagnosis [3]. The survival rate of HNSCC has remained unchanged in the last three decades, partly because rare biomarkers predict the clinical prognosis of this disease and therapeutic effects. As such, there is an urgent need to discover effective regulators associated with the disease stage and clinical prognosis of HNSCC.

Studies over the past two decades have provided much information about large-scale genome sequencing and transcriptome profiling. These studies have identified numerous RNAs, microRNAs (miRNAs), long non-coding RNAs (lncRNAs), and short non-coding RNAs (sncRNAs) [4, 5]. IncRNAs serve as transcription factors or enhancers and participate in various critical biological processes such as chromatin remodelling, histone modification, and genomic imprinting [6-8]. With accumulating evidence of its crucial roles in cancer metastasis, the role of IncRNA in cancers has recently gained attention [9]. Studies have revealed that miRNAs repress target gene expression via shared partial complementarity miRNA response elements (MREs) with the mRNA sequence [10], and lncRNAs function as competing endogenous RNAs (ceRNAs) that interact with mRNAs, acting as miRNA sponges, to restrain miRNA function by competing for MREs [11-13]. The aberrant expression of core IncRNAs in the ceRNA network has a significant impact on network crosstalk and disrupts miRNA-mediated IncRNA/mRNA ceRNA crosstalk interactions, contributing to the initialisation and development of cancers $[12,14]$. The discovery of these interactions provided an important opportunity to advance our understanding of cancer therapy. Based on this theory, IncRNA-miRNA-mRNA ceRNA networks have been identified in ovarian cancer, pancreatic cancer, and lung adenocarcinoma [15-17]. However, there are few comprehensive analyses of HNSCC-associated IncRNAs and miRNAs based on a ceRNA network in the context of a large sample size.

The Cancer Genome Atlas (TCGA), a large integrated collection of clinicopathological information and gene sequencing data across 33 human cancer types, allows for systematic cancer research to improve the prevention, diagnosis and treatment of cancer. In this study, we analysed and compared RNA sequencing data between 502 tumour tissues and 44 normal tissues, and miRNA sequencing data between 525 tumour tissues and 44 normal tissues of TCGA-HNSCC. The miRDB, miRTarBase, and miRcode databases were used to identify the core DEmiRNAs and DElncRNAs, as well as DEmiRNA-DEmRNA interactions. In total, 2, 023 mRNAs, 1, 048 lncRNAs and 83 DEmiRNAs were identified as being aberrantly expressed. To illuminate potential crosstalk in the IncRNA-miRNA-mRNA ceRNA network, we further predicted interactions between miRNAs and IncRNAs. Ultimately, we constructed a ceRNA network that involved 54 lncRNAs, eight miRNAs, and 16 mRNAs.

\section{Materials and Methods}

\section{Acquisition and analysis of expression profiles}

The miRNA expression profiles (44 normal tissues, 525 tumour tissues) and RNA expression profiles (44 normal tissues, 502 tumour tissues) were downloaded from the TCGA website (https://portal.gdc. cancer.gov/). The IncRNA and mRNA expression profiles were then separated from the RNA sequencing data.

After tumour data and normal tissue data were combined, the raw count data were processed with edgeR, an R/Bioconductor package for analysis of differential gene expression profiles with biological replication. Fold-change (tumour/normal) was used to measure the change degree. For $p$-values, the false discovery rate (FDR) was applied to determine the significance threshold for multiple tests. Genes with an absolute $\mid \log 2$ FoldChange $\mid>2$ and an FDR $<0.01$ were considered significant. 


\section{Cellular Physiology Cell Physiol Biochem 2018;50:332-341 \begin{tabular}{ll|l} 
DOI: 10.1159/000494009 218 & $\begin{array}{l}\text { O 2018 The Author(s). Published by S. Karger AG, Basel } \\
\text { www.karger.com/cpb }\end{array}$
\end{tabular}

\section{Regulatory relationship between DEmiRNAs and DElncRNAs}

Putative interactions of miRNA-IncRNA were predicted by the miRcode database (http://www. mircode.org/), which includes 1, 329, 071 interactions between miRNAs and IncRNAs, and allows whole transcriptome human miRNA target predictions based on a comprehensive GENCODE IncRNA annotation.

Weighted gene co-expression network analysis (WGCNA)

WGCNA is an algorithm used in gene co-expression network identification. This algorithm is based on high-throughput mRNA gene expression profiles and has been widely used to determine changes in various diseases [18]. To identify the interactions between DEmRNAs and co-expression modules, we applied WGCNA with a power cut-off threshold of 12 and a module size cut-off 20.

\section{Functional annotation and protein-protein interaction (PPI)}

GO enrichment analysis is widely used in functional annotation. A functional synergistic relationship is defined as when more than one co-regulated target gene is significantly enriched in GO functional terms. To further understand each term, clusterProfiler (http://bioconductor.org/packages/release/bioc/ html/clusterProfiler.html) with a strict cut-off of FDR $<0.05$ was applied to depict the biological process enrichment [19]. Based on a projection at a specific level of the GO corpus, the clusterProfiler package offers a classification method to classify genes, and calculates the enrichment test for GO terms and KEGG pathways based on a hypergeometric distribution. Encoded by multiple genes, tightly interacted groups of proteins can execute complex cellular functions. STRING (Search Tool for the Retrieval of Interacting Genes, http://string-db.org/) [20] was applied to identify PPIs with a high confidence interaction score of 0.7 (the turquoise module) and 0.4 (other modules). In addition, Cytoscape software was used to visualise the PPI networks (http://cytoscape.org/) [21].

\section{Target mRNAs of DEmiRNAs}

DEmiRNA-targeted mRNAs were retrieved from miRTarBase (http://mirtarbase.mbc.nctu.edu.tw/), TargetScan (http://www.targetscan.org/vert_71/) and miRDB (http://www.mirdb.org/). As a database, miRTarBase has accumulated more than 360, 000 miRNA-target interactions (MTIs), which were collected by manually surveying pertinent literature after systematic, natural language processing of the text to filter research articles related to the functional study of miRNAs [22]. TargetScan predicts the biological targets of miRNAs by searching for the presence of conserved 8mer, $7 \mathrm{mer}$, and $6 \mathrm{mer}$ sites that match the seed region of each miRNA, as an option, predictions with only poorly conserved sites are also provided [23]. miRDB is an online database for miRNA target prediction and functional annotations, genome-wide miRNA target prediction was performed with the MirTarget algorithm [24].

\section{Construction of the ceRNA network}

The IncRNA-associated ceRNA network in HNSCC was constructed based on the following procedure: first, miRNA-lncRNA interactions were evaluated using the miRcode database, as mentioned above. Next, miRNA-targeted mRNAs were retrieved from miRTarBase, TargetScan and miRDB, and targets not included in the DEmRNAs were excluded. The results were then visualised using Cytoscape 3.4.0. The main procedure of the method is shown in Fig. 1.

\section{Comparison of gene expression levels}

Gene Expression Profiling Interactive Analysis (GEPIA, http://gepia.cancer-pku.cn/) is a web-based server that provides customisable functions, such as tumour/normal differential expression analysis, profiling according to cancer type or pathological stage, patient survival analysis, detection of similar genes, correlation analysis, and dimensionality reduction analysis [25]. 
Fig. 1. Main when constructing the ceRNA network.

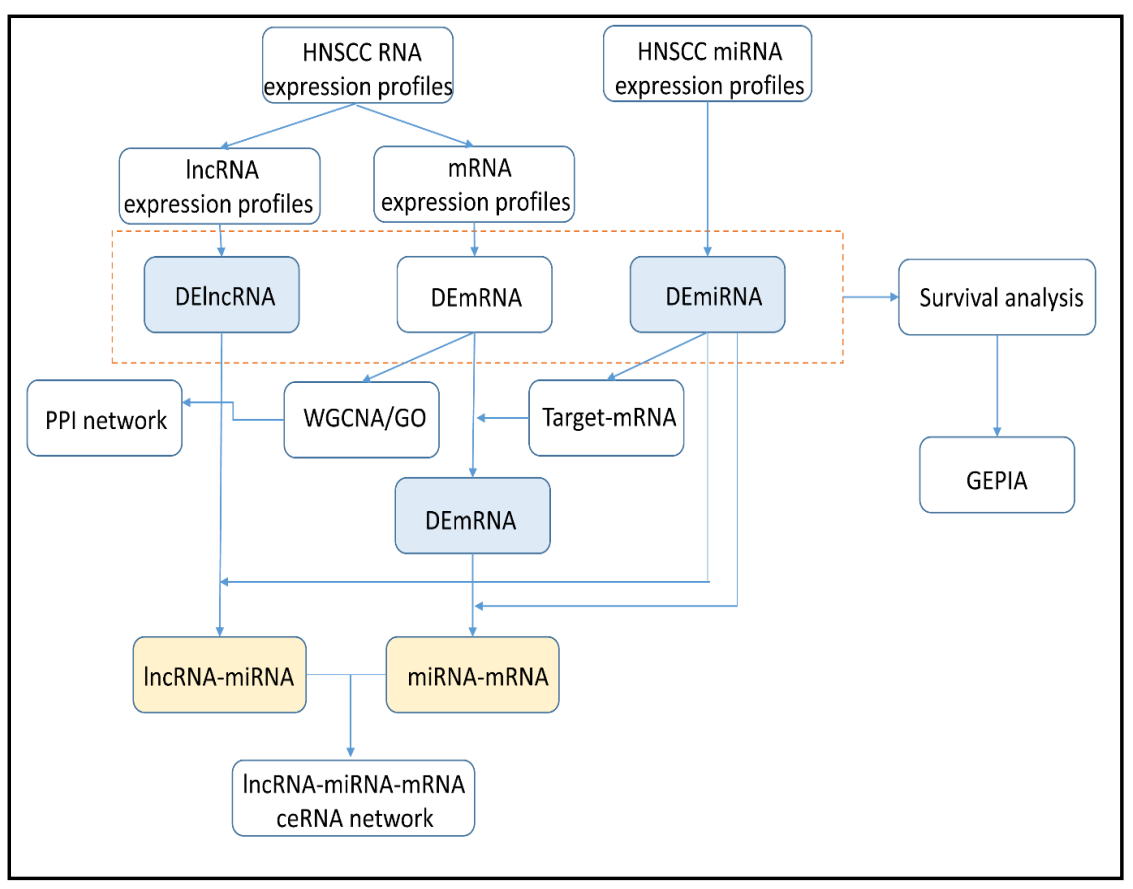

\section{Results}

DEmRNAs, DElncRNAs and DEmiRNAs in HNSCC

The expression profiles of HNSCC patients and their corresponding clinical information were obtained from the TCGA database, and mRNAs and IncRNAs were separated from the RNA expression profiles. Following the differential expression analysis, 2, 023 mRNAs and 1, 048 lncRNAs were identified as being aberrantly expressed (|log2FoldChange| $>2$ and FDR < 0.01). Of these, 870 mRNAs and 757 lncRNAs were up-regulated and 1, 155 mRNAs and 291 IncRNAs were downregulated in tumour tissues compared with normal tissues.

To construct an IncRNA-miRNA-mRNA ceRNA network, we analysed the miRNA expression profiles of 525 TCGA-HNSCC tumour tissues and 44 normal tissues. A total of 82 DEmiRNAs (44 up- and 38 down-regulated) were differentially expressed.

\section{WGCNA analysis, PPI and functional annotation of the modules}

To illustrate the functional clusters of DEmRNAs in HNSCC patients, gene co-expression network analysis of 2, 023 DEmRNAs was carried out using WGCNA. Ten colour modules (non-clustering DEmRNAs are represented in gray) are shown in Fig. 2. As illustrated in Supp. Fig. 1D, the turquoise module contained 353 nodes and 600 edges. Some genes were central to PPI including TNNI1, TNNI2, TNNC1, TNNC2, and TNNT3. Most proteins in this module are involved in muscle cell development and contraction, myofibrils, contractile fibres, actin binding, and the structural constituents of muscle (Fig. 3D).

The most significant functions of the proteins in the blue module were microtubulebased movement, cilium organisation, and cilium assembly in the ontology of biological processes (BP), ciliary parts, cytoplasmic region and axoneme and motor activity in the ontology of the cellular component (CC), microtubule motor activity, and ATP-dependent microtubule motor activity in the ontology of molecular function (MF) (Fig. 3C). Some genes such as DNALI1 and RSPH4A were at the core of the network (Supp. Fig. 1B - for all supplemental material see www.karger.com/10.1159/000494009/).

The average node degree in the brown module was 1.25; proteins in this module were related to the pathway of salivary secretion and collecting duct acid secretions. GNG7, EDN3, and CST4 were core nodes in the PPI of this module (Supp. Fig. 1C). We found that most of the proteins are related to the negative regulation of endopeptidase and peptidase activity, 
Fig. 2. Cluster dendrogram and color display of the coexpression network modules produced by average linkage hierarchical clustering of genes based on topological overlaps in the DEmRNAs. Each branch in the dendrogram is a line that represents a single gene. Its height indicates the Euclidean distance. Each color indicates a single module that contained closely conserved genes. The area occupied by each color is proportional to the number of genes within the respective module.

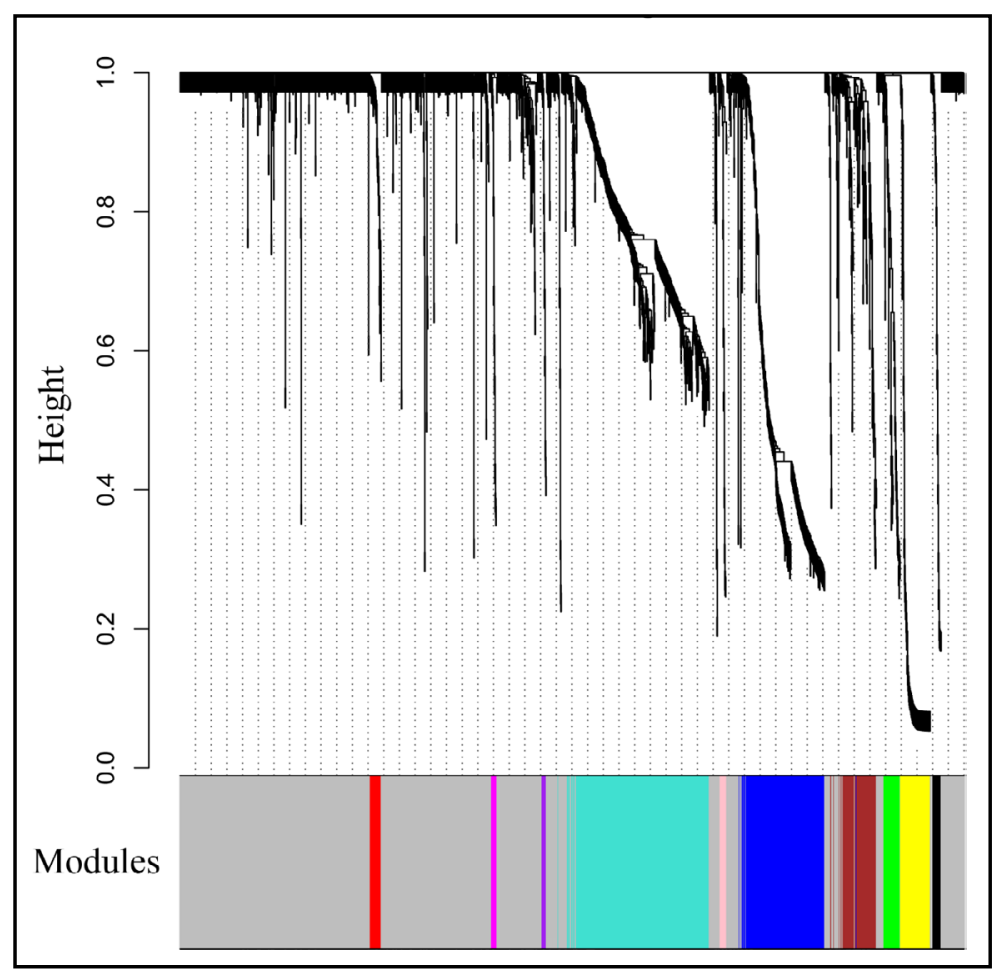

the detection of chemical stimuli involved in the sensory perception of taste, the sensory perception of taste, enzyme inhibitors, endopeptidase inhibitors, and endopeptidase regulator activity (Fig. 3B).

The yellow module contained 75 nodes and 101 edges (Supp. Fig. 1D). In this module, 77 proteins were enriched and involved in keratinisation, keratinocyte differentiation, epidermal cell differentiation, skin development, epidermis development, intermediate filaments, and the intermediate filament cytoskeleton (Fig. 3A).

\section{Establish and analysis of the ceRNA network in HNSCC}

We applied miRcode to search for interactions between miRNAs and lncRNAs. In the network, mRNAs were measured by targets of DEmiRNAs from miRTarBase, TargetScan, and miRDB, and targets not included in DEmRNAs were excluded. Ultimately, the ceRNA network was constructed using eight miRNAs, 53 lncRNAs, and 16 mRNAs (Supp. Fig. 2). Most of the mRNAs in the network have been reported to be cancer-associated genes, such as stanniocalcin 2 (STC2) [26], tropomyosin 2 (TPM2) [27], and transforming growth factor beta receptor 3 (TGFBR3) [28]. Some of the miRNAs in the network had no target mRNAs, such as has-mir-310b, has-mir-31, and has-mir-375. Three miRNAs (has-mir-206, has-mir-210, and has-mir-503) targeted a single mRNA. Has-mir-211 had the most target mRNAs. The target mRNAs of has-mir-195 were mostly up-regulated.

Survival and expression level analysis of mRNAs, IncRNAs and miRNAs in the network

Kaplan-Meier curve analysis was used to investigate overall survival for the DElncRNAs, DEmRNAs, and DEmiRNAs as ceRNA counterparts. miRNA has-mir-206, mRNA STC2, and five DElncRNAs (ABCA9-AS1, ANO1-AS2, HOTTIP, LINC00052, and LINC00460) were negatively associated with worse overall survival $(p<0.05)$ for HNSCC patients. By contrast, four DElncRNA (HCG22, MIAT, MUC19, and ZFY-AS1) prolonged patient survival time $(p<0.05$, Fig. 4).

Next, we applied GEPIA to verify the correlations with overall survival and to detect the difference in expression levels of IncRNAs between tumour and normal tissues. The result indicated that LINC00460 and HCG22 were aberrantly expressed and closely associated 


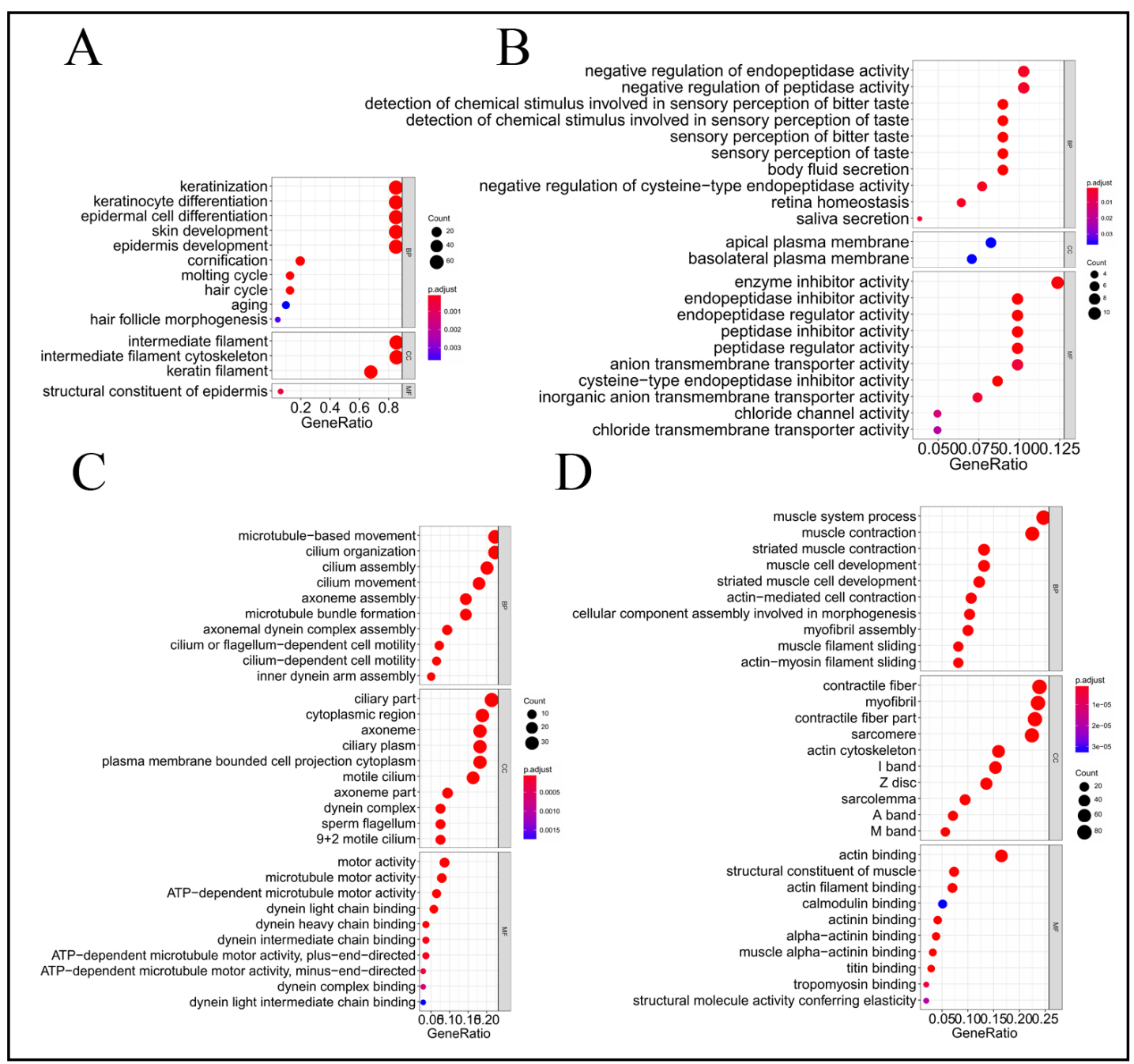

Fig. 3. GO enrichment of gene clusters in the yellow (A), brown (B), blue (C), and turquoise (D) modules. The coloured dots represent term enrichment: blue indicates low enrichment, red indicates high enrichment. The sizes of the dots represent the number of genes in each GO category.

with overall survival (Fig. 4). LINC00460 exhibited higher expression in 519 tumour tissues than in 44 normal tissues, while HCG22 showed the opposite pattern. Kaplan-Meier survival curves indicated that IncRNA HCG22 was positively correlated with overall survival, whereas LINC00460 was negatively associated with overall survival.

\section{Characteristics of STC2 in HNSCC}

Next, we evaluated the expression of STC2 in tumour tissues compared to normal tissues. As depicted in Fig. 4A, STC2 transcript levels were significantly higher in tumour tissues than in normal tissues. Kaplan-Meier survival curves indicated that STC2 is positively correlated with overall survival (Fig. 4B). We also examined the expression of STC2 in different major pathological stages of HNSCC; the results indicated that STC2 was slightly upregulated at stages 1, 2, 3, and 4 (Fig. 4C). 
A
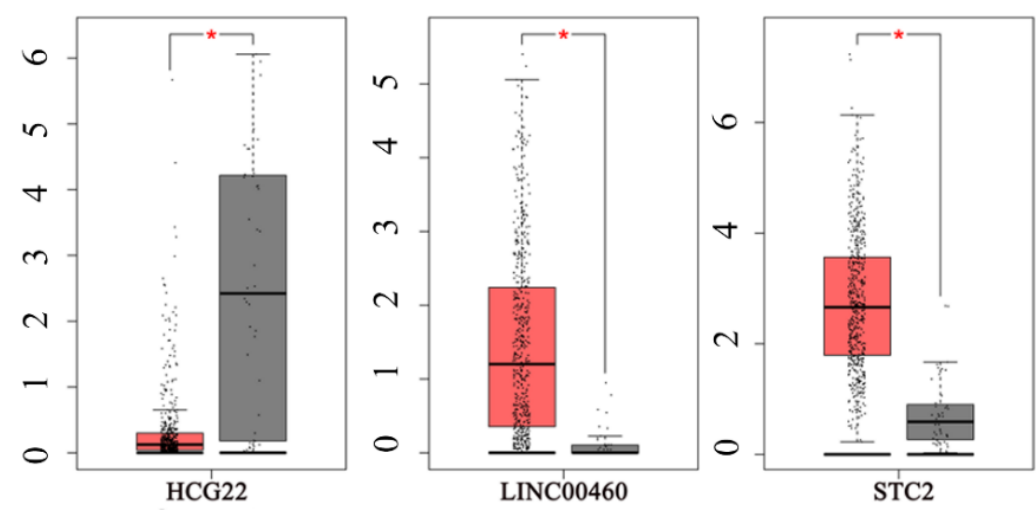

B

$$
(\operatorname{num}(\mathrm{T})=519 ; \operatorname{num}(\mathrm{N})=44)
$$
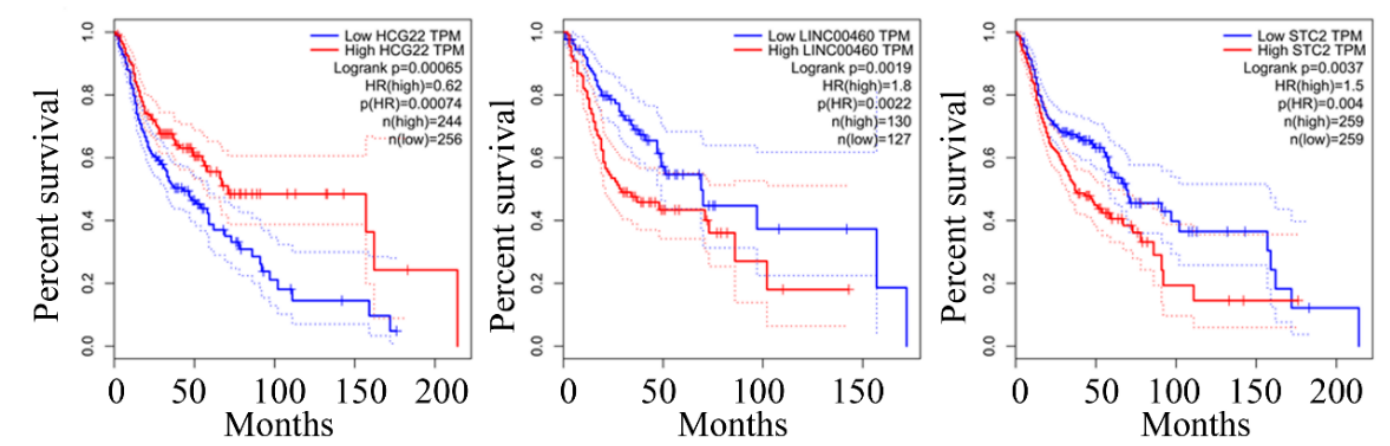

C

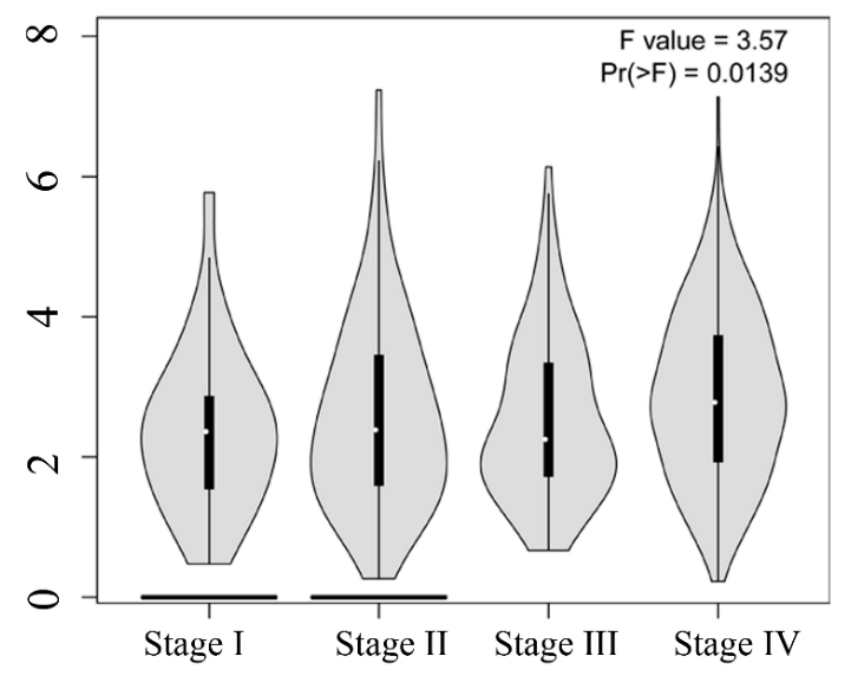

Fig. 4. Expression of HCG22, LINC00460, and STC2 in HNSCC and normal tissues from GEPIA. (A) Expression was examined in 519 HNSCC tissues (T) and 44 normal tissues $(\mathrm{N}$ ) from GEPIA. The axis units are $\log 2(\mathrm{TPM}+1)$. (B) Kaplan-Meier curve analysis of HCG22, LINC00460, and STC2 for the overall survival in HNSCC patients. (C) Violin plot showing STC2 expression in different major pathological stages of HNSCC. 


\section{Cellular Physiology Cell Physiol Biochem 2018;50:332-341 \begin{tabular}{cc|c|} 
DOI: 10.1159/000494009 & O 2018 The Author(s). Published by S. Karger AG, Basel \\
www.karger.com/cpb
\end{tabular} \\ Zhao et al.: Non-Coding RNAs and MicroRNAs Medicate Cancer Cell Network}

\section{Discussion}

A growing number of studies have lent support to the ceRNA hypothesis, a novel gene regulation layer, in which IncRNAs serve as miRNA sponges and participate in various biological processes and their expression is associated with many complicated disease phenotypes, including cancers [29-32]. Research studies have demonstrated the critical role of IncRNAs [33, 34], and have shown that the aberrant expression of IncRNAs contributes to extensive variation in gene expression via the IncRNA-mediated ceRNA regulatory network. However, the molecular mechanism of the ceRNA regulatory network is unclear in HNSCC. In the current study, we constructed an IncRNA-related ceRNA network by integrating the TCGA-HNSCC expression profiles, which will shed light on how lncRNAs and miRNAs are involved in HNSCC.

The HCG22 transcript, which encodes a novel mucin protein, is stimulated by interleukin (IL)-1, inhibited by triamcinolone acetate and tumour growth factor- $\beta$, and is expressed in trabecular meshwork cells. Our analysis suggests a potential mechanism by which HCG22 competes with miR-195 to regulate the expression of TPM2, SH3BGRL2, HOXA10, TGFBR3, ALOX12, and TMEM100. Manipulated by the transcriptional co-activator CBP/ P300 via histone acetylation, LINC00460 was overexpressed in most tumour tissues. In vitro experiments revealed that LINC00460 depletion suppressed oesophageal squamous cell carcinoma (OSCC) growth by regulating cell proliferation, the cell cycle and apoptosis [35]. On reviewing published research, we noticed that LINC00460 exerts its oncogenic function in large part via sponging miR-149-5p and subsequently initiating the IL-6 signalling pathway in nasopharyngeal carcinoma [36]. Our analysis revealed a novel potential mechanism whereby LINC00460 competes with miR-206 to regulate the expression of STC2, or competes with miR-503 to regulate the expression of GREM2. STC2 is correlated with a poor prognosis in OSCC via lymph node metastases [37], and miR-206 is a muscle-specific miRNA that participate in a series of cellular activities including skeletal muscle development and tumourigenesis $[38,39]$. IncRNA is conserved in various cancers to an extent, which suggested that IncRNA-related ceRNA regulation is a fundamental gene regulation layer in tumourigenesis.

According to the modules produced by WGCNA, many core genes from the turquoise module, such as CHRDL1, GPD1L, KLHL40, TMEM100, and TPM2, as well as GREM2, HOXA10, and SH3BGRL2 from the grey module, were considered important target proteins. HOXA10, SH3BGRL2, TMEM100, TPM2, and HCG22 are target genes of miR-195. Previous studies have revealed that miR195 promotes colorectal cancer cell (SW620) apoptosis by down-regulating Siah-1S [40]. Moreover, the overexpression of miR-195 suppressed breast cancer cell proliferation and invasion [41]. Therefore, we believe that miR-195 may also be critical to HNSCC.

\section{Conclusion}

In conclusion, we performed a comprehensive analysis of IncRNA-mediated ceRNA interplay based on the IncRNA and RNA expression profiles of HNSCC, which for the first time enabled an overview and analysis of the role of a ceRNA regulatory network of HNSCC at a system-wide level. By comprehensively analysing the lncRNA-related ceRNA network, some novel and crucial characters of HNSCC were revealed. These findings shed some light on the regulation mechanism in HNSCC and will be beneficial for mining potential therapeutic targets or candidate diagnostic biomarkers. 


\section{Cellular Physiology Cell Physiol Biochem 2018;50:332-341

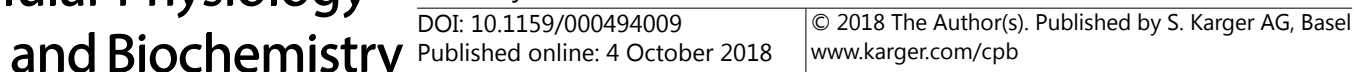

Zhao et al.: Non-Coding RNAs and MicroRNAs Medicate Cancer Cell Network

\section{Acknowledgements}

This work is supported by Fundamental Research Funds for the Central Universities (No. 2015ZCQ-SW-06, NO. BLX2015-23).

\section{Disclosure Statement}

The authors declare that no conflict of interests exist.

\section{References}

1 The Cancer Genome Atlas Network: Network CGA: Comprehensive genomic characterization of head and neck squamous cell carcinomas. Nature 2015;517:576.

- Xu CZ, Jiang C, Wu Q Liu L, Yan X, Shi R: A Feed-Forward Regulatory Loop between HuR and the Long Noncoding RNA HOTAIR Promotes Head and Neck Squamous Cell Carcinoma Progression and Metastasis. Cell Physiol Biochem 2016;40:1039-1051.

3 Li S, Yang X, Wang P, Ran X: The effects of GLUT1 on the survival of head and neck squamous cell carcinoma. Cell Physiol Biochem 2013;32:624-634.

4 Carninci P, Kasukawa T, Katayama S, Gough J, Frith MC, Maeda N, Oyama R, Ravasi T, Lenhard B, Wells C: The transcriptional landscape of the mammalian genome. Science 2005;309:1559-1563.

5 Birney E, Stamatoyannopoulos JA, Dutta A, Guigó R, Gingeras TR, Margulies EH, Weng Z, Snyder M, Dermitzakis ET, Thurman RE: Identification and analysis of functional elements in $1 \%$ of the human genome by the ENCODE pilot project. Nature 2007;447:799-816.

-6 Shi X, Sun M, Wu Y, Yao Y, Liu H, Wu G, Yuan D, Song Y: Post-transcriptional regulation of long noncoding RNAs in cancer. Tumour Biol 2015;36:503.

-7 Kornienko AE, Guenzl PM, Barlow DP, Pauler FM: Gene regulation by the act of long non-coding RNA transcription. BMC Biol 2013;11:59.

8 Fatica A, Bozzoni I: Long non-coding RNAs: new players in cell differentiation and development. Nat Rev Genet 2014;15:7-21.

-9 Prensner JR, Chinnaiyan AM: The emergence of lncRNAs in cancer biology. Cancer Discov 2011;1:391.

10 Ye W, Lv Q, Wong CKA, Hu S, Fu C, Hua Z, Cai G, Li G, Yang BB, Zhang Y: The effect of central loops in miRNA:MRE duplexes on the efficiency of miRNA-mediated gene regulation. PloS One 2008;3:e1719.

11 Tay Y, Rinn J, Pandolfi PP: The multilayered complexity of ceRNA crosstalk and competition. Nature 2014;505:344-352.

12 Tan JY, Sirey T, Honti F, Graham B, Piovesan A, Merkenschlager M, Webber C, Ponting CP, Marques AC: Extensive microRNA-mediated crosstalk between IncRNAs and mRNAs in mouse embryonic stem cells. Genome Res 2015;25:655.

13 Salmena L, Poliseno L, Tay Y, Kats L, Pandolfi PP: A ceRNA hypothesis: the Rosetta stone of a hidden RNA language? Cell 2011;146:353-358.

-14 Karreth FA, Pandolfi PP: ceRNA Cross-Talk in Cancer: When ce-bling Rivalries Go Awry. Cancer Discov 2013;3:1113.

15 Zhou M, Wang X, Shi H, Cheng L, Wang Z, Zhao H, Yang L, Sun J: Characterization of long non-coding RNAassociated ceRNA network to reveal potential prognostic lncRNA biomarkers in human ovarian cancer. Oncotarget 2016;7:12598-12611.

16 Zhou M, Diao Z, Yue X, Chen Y, Zhao H, Cheng L, Sun J: Construction and analysis of dysregulated IncRNAassociated ceRNA network identified novel lncRNA biomarkers for early diagnosis of human pancreatic cancer. Oncotarget 2016;7:56383-56394.

17 Sui J, Li YH, Zhang YQ, Li CY, Shen X, Yao WZ, Peng H, Hong WW, Yin LH, Pu YP, Liang GY: Integrated analysis of long non-coding RNA-associated ceRNA network reveals potential lncRNA biomarkers in human lung adenocarcinoma. Int J Oncol 2016;49:2023.

18 Langfelder P, Horvath S: WGCNA: an R package for weighted gene co-expression network analysis. BMC Bioinformatics 2008:9:559. 


\section{Cellular Physiology Cell Physiol Biochem 2018;50:332-341

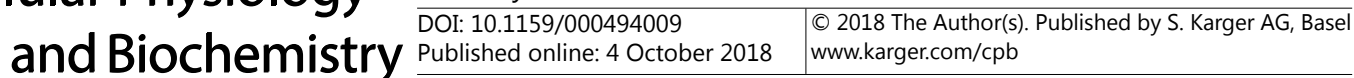

19 Yu G, Wang LG, Han Y, He QY: clusterProfiler: an R Package for Comparing Biological Themes Among Gene Clusters. OMICS 2012;16:284-287.

-20 Franceschini A, Szklarczyk D, Frankild S, Kuhn M, Simonovic M, Roth A, Lin J, Minguez P, Bork P, Von Mering C, Jensen LJ: STRING v9.1: protein-protein interaction networks, with increased coverage and integration. Nucleic Acids Res 2013;41:D808-D815.

21 Kohl M, Wiese S, Warscheid B: Cytoscape: software for visualization and analysis of biological networks. Methods Mol Biol 2011;696:291-303.

$>22$ Chou CH, Shrestha S, Yang CD, Chang NW, Lin YL, Liao KW, Huang WC, Sun TH, Tu SJ, Lee WH, Chiew MY, Tai CS, Wei TY, Tsai TR, Huang HT, Wang CY, Wu HY, Ho SY, Chen PR, Chuang CH: miRTarBase update 2018: a resource for experimentally validated microRNA-target interactions. Nucleic Acids Res 2017;46:D296-D302.

23 Lewis BP, Burge CB, Bartel DP: Conserved seed pairing, often flanked by adenosines, indicates that thousands of human genes are microRNA targets. Cell 2005;120:15.

24 Wang X, El Naqa IM: Prediction of both conserved and nonconserved microRNA targets in animals. Bioinformatics 2008;24:325.

25 Tang Z, Li C, Kang B, Gao G, Li C, Zhang Z: GEPIA: a web server for cancer and normal gene expression profiling and interactive analyses. Nucleic Acids Res 2017;45.

26 Wu J, Lai M, Shao C, Wang J, Wei JJ: STC2 overexpression mediated by HMGA2 is a biomarker for aggressiveness of high-grade serous ovarian cancer. Oncol Rep 2015;34:1494.

27 Zhang J, Zhang J, Xu S, Zhang X, Wang P, Wu H, Xia B, Zhang G, Lei B, Wan L, Zhang D, Pang D: HypoxiaInduced TPM2 Methylation is Associated with Chemoresistance and Poor Prognosis in Breast Cancer. Cell Physiol Biochem 2018;45:692.

28 Sharifi N, Hurt EM, Kawasaki BT, Farrar WL: TGFBR3 loss and consequences in prostate cancer. Prostate 2007;67:301-311.

29 Cheetham SW, Gruhl F, Mattick JS, Dinger ME: Long noncoding RNAs and the genetics of cancer. Br J Cancer 2013;108:2419.

-30 Zhou M, Wang X, Li J, Hao D, Wang Z, Shi H, Han L, Zhou H, Sun J: Prioritizing candidate disease-related long non-coding RNAs by walking on the heterogeneous lncRNA and disease network. Mol Biosyst 2015;11:760.

-31 Sun J, Shi H, Wang Z, Zhang C, Liu L, Wang L, He W, Hao D, Liu S, Zhou M: Inferring novel lncRNA-disease associations based on a random walk model of a IncRNA functional similarity network. Mol Biosyst 2014;10:2074-2081.

-32 Sun J, Yan J, Yuan X, Yang R, Dan T, Wang X, Kong G, Gao S: A computationally constructed ceRNA interaction network based on a comparison of the SHEE and SHEEC cell lines. Cell Mol Biol Lett 2016;21:21.

33 He X, Zhang J: Why do hubs tend to be essential in protein networks? PLoS Genet 2006;2:e88.

-34 Gursoy A, Keskin 0, Nussinov R: Topological properties of protein interaction networks from a structural perspective. Biochem Soc Trans 2008;36:1398.

-35 Liang Y, Wu Y, Chen X, Zhang S, Wang K, Guan X, Yang K, Li J, Bai Y: A novel long noncoding RNA linc00460 up-regulated by CBP/P300 promotes carcinogenesis in esophageal squamous cell carcinoma. Biosci Rep 2017;37:BSR20171019.

-36 Kong YG, Cui M, Chen SM, Xu Y, Xu Y, Tao ZZ: LncRNA-LINC00460 facilitates nasopharyngeal carcinoma tumorigenesis through sponging miR-149-5p to up-regulate IL6. Gene 2018;639:77.

-37 Kita Y, Mimori K, Iwatsuki M, Yokobori T, Ieta K, Tanaka F, Ishii H, Okumura H, Natsugoe S, Mori M: STC2: a predictive marker for lymph node metastasis in esophageal squamous-cell carcinoma. Ann Surg Oncol 2011;18:261-272.

38 Jin P, Gu W, Lai Y, Zheng W, Zhou Q, Wu X: The Circulating MicroRNA-206 Level Predicts the Severity of Pulmonary Hypertension in Patients with Left Heart Diseases. Cell Physiol Biochem 2017;41:2150.

-39 Wang R, Hu Y, Song G, Hao CJ, Cui Y, Xia HF, Ma X: MiR-206 regulates neural cells proliferation and apoptosis via Otx2. Cell Physiol Biochem 2012;29:381-390.

40 Yu G, Wang T, Cheng Z, Lv W: MiR195 downregulating Siah-1S level to inhibit cell growth and promote cell apoptosis in colorectal cancer cells SW620. Int J Clin Exp Pathol 2016;9:5937-5943.

41 Li D, Zhao Y, Liu C, Chen X, Qi Y, Jiang Y, Zou C, Zhang X, Liu S, Wang X, Zhao D, Sun Q, Zeng Z, Dress A, Lin MC, Kung HF, Rui H, Liu LZ, Mao F, Jiang BH, Lai L: Analysis of MiR-195 and MiR-497 expression, regulation and role in breast cancer. Clin Cancer Res 2011;17:1722-1730. 